\title{
Detection of Cataract Through Feature Extraction by the Novel Angular Binary Pattern (NABP) and Classification by Kernel Based Convolutional Neural Networks
}

\author{
A Sirajudeen ( $\nabla$ sirajudeenphd@gmail.com ) \\ Aurora's Scientific Technological and Research Academy https://orcid.org/0000-0003-4108-8072 \\ Anuradha balasubramaniam \\ Sri Eshwar College of Engineering \\ S Karthikeyan \\ Malla Reddy Engineering College and Management Sciences
}

\section{Research Article}

Keywords: Cataract, Novel Angular Binary Pattern (NABP), Kernel Based Convolutional Neural Networks, Color features, Texture features and Shape features.

Posted Date: April 5th, 2021

DOI: https://doi.org/10.21203/rs.3.rs-383419/v1

License: (c) (1) This work is licensed under a Creative Commons Attribution 4.0 International License.

Read Full License 


\title{
Detection of Cataract Through Feature Extraction by the Novel Angular Binary Pattern (NABP) and Classification by Kernel Based Convolutional Neural Networks
}

\author{
${ }^{* 1}$ A. Sirajudeen, ${ }^{2}$ Anuradha balasubramaniam, ${ }^{3}$ Dr.S.Karthikeyan \\ ${ }^{1}$ Professor, Aurora's Scientific \& Technological Institute, Ghatkesar, India. \\ ${ }^{2}$ Assistant professor, Sri Eshwar College Of Engineering, Coimbatore, India. \\ ${ }^{3}$ Associate Professor, Dept of ECE, Malla Reddy Engineering College and Management Sciences, Medchal, India. \\ Corresponding Author's Id: sirajudeenphd@gmail.com
}

\begin{abstract}
Cataract is a condition of the opacity in the lenticular regions, which usually results in bad visual interpretation of the viewed object or any entity. Hence the timely detection of cataract is considered to be significant and can even contribute in the prevention from loss of fight that might occur if the cataract is left untreated. In this paper, detection of cataract disease is carried out based on the image processing technique. Color features, texture features and shape features are extracted separately. This study proposed a Novel Angular Binary Pattern (NABP) for the extraction of texture features. And after the extraction of features, the images are subjected to classification through the implementation of the proposed novel Kernel Based Convolutional Neural Networks. Results are obtained separately for all the three types of features. A comparison is carried out for the proposed work with existing works and based on the results obtained it can be seen that the proposed work comes up with the enhanced results than the traditional methods.
\end{abstract}

Index Terms - Cataract, Novel Angular Binary Pattern (NABP), Kernel Based Convolutional Neural Networks, Color features, Texture features and Shape features.

\section{INTRODUCTION}

Cataract is a condition of the opacity in the lenticular regions, which usually results in with bad visual interpretation of the viewed object or any entity. More than 40,000,000 people are expected to suffer with blindness within the next decade as per the recent reports published WHO-World Health Organization. In general, WHO declare that around half of the blind patients were found to have the cataracts symptoms or severities. Usually, may patients tend to postpone the cataract operation for couple of years after being detected with cataract, but the patients should realize that the severity increases as the time goes on [1]. Also, it was necessary to improve the preidentification phase in the cataract abnormalities because of the sensitiveness of the lens wherein the light is being focused onto the region of the retina in the eye for achieving the precise vision. The lens additionally makes the adjustments to the eye focus to vary the rate of vision for viewing both the far way and nearby objects or entities. The protein presence would be making the eye lenses to work precisely, thereby making our vision to be clear. But, as the age goes on, for some people, some proteins particles in the eye unexpectedly combines with other protein particles, thereby, forming the tiny areas of cloud structure in the lens of the eye. This condition was only widely regarded as the cataract wherein the cloud structure keeps on augment itself as the time goes on and finally making it difficult to view the intended object or the entity clearer. Simply, a cataract was nothing but the built up of protein specific cloud contents that makes things difficult to view the objects or entities. As the period goes on, this cataract condition gets severe to cause the blindness of the eye. Thus, proper and timely prevention is better than cure [2].
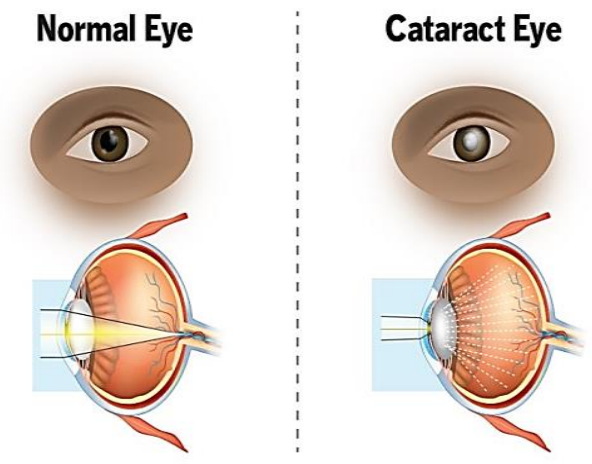

Figure 1. Difference between normal and cataract eye

Based on the reason for the occurrence of cataracts, it can categorized into three common categories like age related cataracts, pediatric cataracts and other secondary type of cataracts that are occurred due to various other reasons. Age related type of cataract is one of the most commonly occurring disease among the grown-ups and especially among the age of 45 to 50 years. The lens become opaque in nature and due to the formation of oxidative stress within the lens. Based on the placement of opaqueness inside the eye lens, age related cataracts were classified into various classifications like nuclear cataract, cortical cataract and posterior sub capsular cataracts. The difference between normal and cataract eye is represented in Figure 1 [3].

The prior detection and diagnosis of cataract disease certainly results in the reduction of sufferings experienced by the cataract affected patients. Mostly, the permanent loss of sight due to the visual impairment caused by cataract can be prevented by the timely detection. Due to the presence of very poor eye related services and shortage of professional eye 
specialists, people in a remote regions could not able to receive appropriate diagnosis for the cataract [4]. Some of the commonly employed approaches utilized for the treatment of cataract involves the usage of slit lamp in the LOCS III (i.e. Lens Opacity Classification System III) for the clinical evaluation, Wisconsin system that performs the grading of cataracts. But these approaches were highly complicated and higher in cost wise for some of the patients in the remote sections. And hence in order to improve the eye care services in remote regions, the process employed for diagnosing the cataracts must be made in a simple manner and costs must be minimized for making it useful for all the cataract affected patients.

Ever since the first implementation of cataract grading methods about 30 years ago, a constant progression is observed in the grading methods till now. It can be employed for both research point of view and also for the medical sector as well. This grading methods are helpful in improving the communication among a patient and an ophthalmologist. The grading systems are very easier to interpret and details regarding the diagnosis, growth of cataract and the significance of treatment can be understood by the patients themselves [5].

In addition, this study [6] have reviewed various techniques for cataract detection. This study has been carried out to detect which system has the ability to detect cataract at its initial stage by classifying them as post cataract and normal cataract. Various techniques reviewed this study include mobile cataract detection system, Tre-training method, fundus image processing, enhanced texture feature and analysis of automatic cataract detection. It has been found that all these techniques afforded satisfactory results in cataract detection. Similarly, this article represents a model to automatically detect as well as classify the cataracts on the basis of AI (Artificial Intelligence) and image processing. The recommended system has been introduced to make the cataract diagnosis easy through prediction.

This article has introduced a wavelet transform integrated with a 2D Log Gabor Wavelet Transform to perform feature selection. This has been followed by classification. The eye images have been classified into early, advanced and normal based on its stages. A comparison has been made amongst the two utilized classifiers namely ANN (Artificial Neural Network) and SVM (Support Vector Machines). It has been summarized that the proposed SVM afforded effective outcomes than ANN. Yet, this model have to be further examined as well as tested against large image datasets to assess the outcomes of the feature extraction and classification techniques [7]. Hence, this study introduced a Novel Angular Binary Pattern (NABP) to perform relevant feature selection. In addition, efficient classification is performed through the proposed novel Kernel Based Convolutional Neural Networks. This introduced method has various merits. The Kernel based CNN parameters like image size, count of hidden layers, size of the kernel, connection tables and skipping factors are flexible to any application. Thus, the proposed methods classify cataract and normal persons in an effective way when compared to traditional methods in terms of accuracy in cataract classification.
The architecture corresponding to kernel based CNN is shown in the below figure 2 .

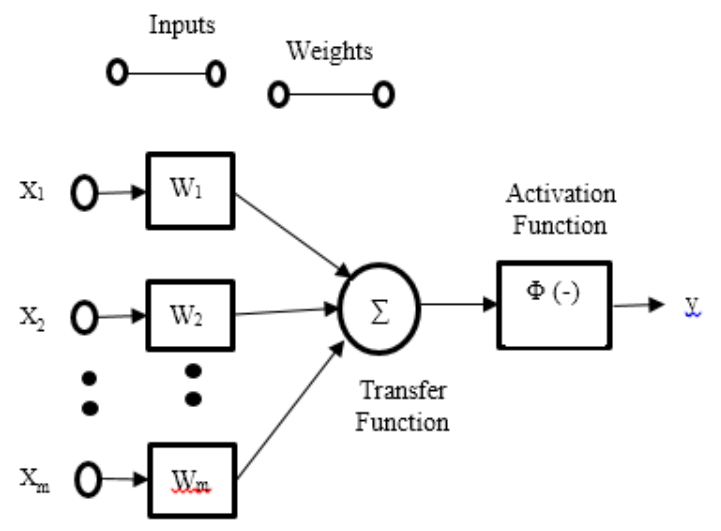

Figure 2. Kernel based CNN architecture

In $\mathrm{CNN}$, the kernel is a filter that is utilized to perform feature extraction from the images. It can be defined as a matrix which moves over the inputs and performs dot product corresponding to the input data sub region. The above architecture consists of activation function and transfer function. Finally, it attains output as the matrix consisting of dot products.

\section{A. Objectives}

- To distinguish between the normal and abnormal images from the input eye images.

- To extract relevant features by the usage of Novel Angular Binary Pattern (NABP).

- To classify the images in an effective way through the usage of Kernel Based Convolutional Neural Networks.

\section{RELATED WORKS}

A brief description of various techniques utilized for the detection of cataract disease among the patients by using the cataract image is given in this section. Then various types of techniques employed for the extraction of features in cataract fundus images are also provided.

This study [8] provided an overall summary for numerous image processing techniques utilized in the evaluation of cataract disease in medical sector. Various approaches employed for the detection of different kinds of eye disease were also enlisted in the work. By analyzing the benefits and shortcomings, the ideal technique for detecting cataracts in the eye along with various types of eye disease could be determined.

Different kinds of methods have been reviewed and employed in this study [9] for identifying the condition of cataract in humans. Various categories of works which discusses about recently utilized images processing techniques like mobile cataract detection system, fundus image processing technique, pre training approach, automatic cataract detection system and enhanced texture feature which were considered to be one among the broadly used techniques in detecting the cataract diseases. 
Histogram has been used and presented in this study [10] for detecting the disease of cataract along with its conjunctivitis. Conjunctivitis is nothing but the condition where the conjunctiva within the eye gets worsened due to some kind of allergy or infection. Initially preprocessing of the cataract images were carried out followed by the extraction of features and classification of the extracted images. Minimum distance classifier was utilized for classifying the images and after that the input cataract images were differentiated into normal eye, cataract affected eye and conjunctivitis affected eye.

Similarly, this paper [11] performed a comparative investigation of various vessel segmentation methods employed for segmenting the retinal images that were obtained from pubic retinal dataset, fundus type of photography technique etc. Various prevailing techniques of both supervised and unsupervised segmentation of blood vessels were reviewed and compared with each other. Every steps of the image processing like preprocessing, post processing, segmentation, feature extraction and results were compared for all the techniques.

Moreover, this article [12] Various imaging modalities implemented for the grading of cataract disease were compared. Basic details of cataract was provides that provides differentiation of normal vision with cataract vision along with the types of cataract diseases were provided.

Likewise, this study [13] The impacts of utilizing the cataracts grading were investigated depending upon the fundus imaging techniques that were employed for the measurement of macular thickness through the SD-OCT (i.e. Spectral Domain Optical Coherence Tomography) and the corresponding SNR (i.e. Signal to Noise Ratio). A sample of 200 patients affected with cataract were considered and macular thickness were measured. The severity of cataract was graded ranging from 1 to 4 depending upon the degree by which the macular was obscured by the condition of cataract in the fundus images. A comparative analysis was made for various factors like thickness of macular, fundus imaging technique and SD-OCT SNR. The errors prevailing within the segmentation of retinal layer were detected by the evaluation of B-scan images in SDOCT.

Accordingly, this paper [14] proposed a system that can be operated by remote for detecting the cataract disease in an automated manner. By this system, the affected persons can able to get appropriate diagnosis from the physicians based on the condition of the affected cataract. This proposed system is employed in the cloud by which the patients can able to access the system at any time. Four stages like preprocessing, feature extraction, classification and grading were present among the proposed remote cataract system.

On the other hand, this paper [15] calculated the average settings of optimal image for the Spectralis OCT among the patients who were affected with cataract and also for the patients who were not affected with cataract.

Similarly, this article [16] recommended a digital image processing technique to identify the cataract present in addition to its severity. Two varied image processing techniques have been employed on a dataset which comprises of eye images possessing varying cataract degrees. The initial strategy utilized a feature extraction on the basis of automated detection methodologies. It takes the image and performed pre- processing and later extracted the information about the intensity. It classified the images as mild cataract, severe cataract or healthy cataract. Analysis has been performed using the plots of histogram. Moreover, contour detection and Hough transform have been utilized to identify the image shapes, inversion utilizing the logical operations have been performed. The cataract percentage has been successfully computed. The algorithms have been compared in terms of accuracy and efficiency which reveals its efficacy. Yet, various types of image-processing methods have to be executed on many datasets as well as tested [17].

In addition, this article [18] utilized CNN (Convolutional Neural Networks) to learn relevant features from the input data directly. The DN (Deconvolution Network) has been applied to examine the way in which CNN illustrated the cataract by individual layers. This article revealed that the vascular information that is mislaid after multi-layer convolution computation has a significant part in cataract grading when compared to global feature collection. This revealed the morphological definition corresponding to the fundus image. The findings exhibited that the proposed method attained effective outcomes.

Likewise, this study [19] explored a cataract detection methodology utilizing CNN. The model has been trained on a dataset consisting of hundred eye images by utilizing transfer learning that have been extracted from google search results corresponding to eye cataract. The study used ImageNet model using CNN classifier. The knowledge has been transferred through transfer learning for training the model. This model attained the capability for classifying the eye images as cataract and normal. The system has been outlined by taking images as its input and accomplished effective classification [20].

Moreover, this paper [21] explored about the current trends corresponding to the automation of cataract detection as well as the necessity of proposing a system to accomplish the detection effectively. This study also viewed the requirement to detect the cataract position to aid in making decisions if there is a need to perform surgery for replacing the lens. Thus, this study intended to introduce a device that has the ability to detect the occurrence, type, position as well as the stage corresponding to the cataract [22]. This study has to be extended further to enhance its efficiency.

\section{Problem Identification:}

It has been observed that the convolution layers considerably decrease the required number of weights. These weights depends on the size of the kernel instead of size of the input which are significant for the images. Further convolutional layers with memory decrease the memory usage and leads to rapid computation. Apart from that the over fitting is significantly less and extracts the data regarding the features of abnormal regions of various patients with various angular orientation. Hence the paper attempt to overcome the problems present in the existing works with NABP and kernel based CNN. 


\section{Proposed Work}

The following describe the concept behind the overall process of the proposed work. The retinal image for cataract detection dataset was used in the presented experimental analysis. Preprocessing was done for noise removal, pixel enhancement, channel separation etc. the study used novel binary angular pattern for the extraction of texture features in accordance with the orientation information of all the single pattern, visual and edge patterns. Additionally, texture features such as wavelet features, GLCM and bit pattern features are also extracted.
Shape features and the color features such as color moments, HSV- Histogram, color Co-occurrence and Color autocorrelogram. Kernel based neural network classifier leads to the enhanced classification of the result followed by performance analysis. The Overall Flow of the proposed work is given in the below figure 3 .

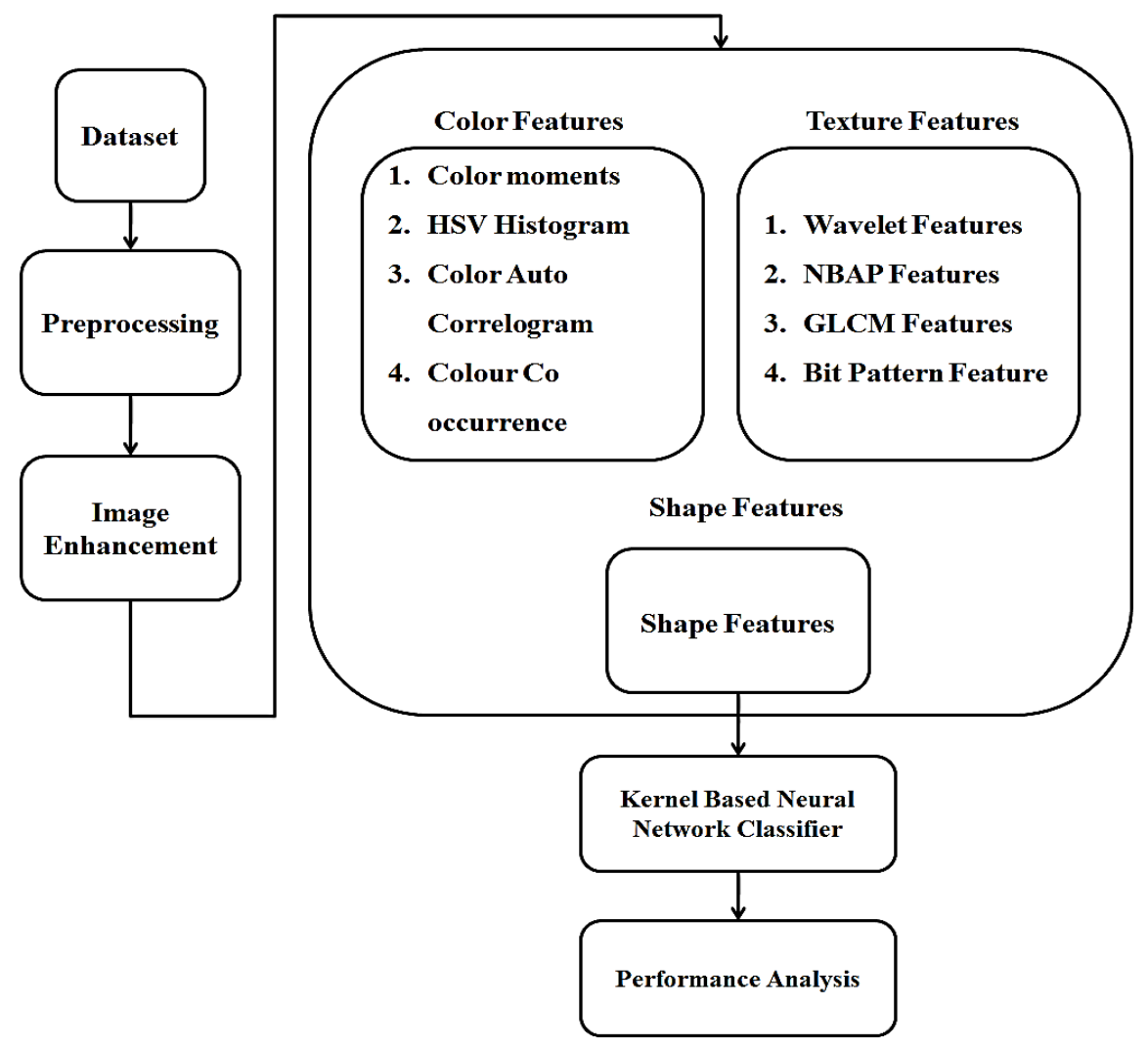

Figure 3. Overall Flow of the proposed work

\subsection{Color Features}

\subsubsection{Color moments}

Color that is distributed within an image can be characterized through the measure of color moments in such a manner that the central moments can able to describe the probability distribution in a unique manner. This measures are employed for color indexing reasons as it determines the similarity between two different images depending upon the color of the images. Normally digital images along with the calculated attributes in a dataset is compared with a particular image for determining and extracting a similar image.

\subsubsection{HSV Histogram}

$$
\begin{gathered}
H=\left\{\begin{array}{c}
60\left(\frac{H-C}{\partial}\right) \text { if } m x=S \\
60\left(\frac{C-S}{\partial}+2\right) \text { if } m x=H \\
60\left(\frac{S-H}{\partial}+4\right) \text { if } m x=C \\
\text { not defined if } m x=0
\end{array}\right. \\
S=\left\{\begin{array}{c}
\frac{m x-m n}{m x} \text { if } m x \text { is not equal to zero } \\
0 \quad \text { if } m x \text { is equal to zero } \\
V=m x
\end{array}\right.
\end{gathered}
$$

Where $\partial(\mathrm{mx}-\mathrm{mn}), \mathrm{mx}=\operatorname{maximum}(\mathrm{S}, \mathrm{H}, \mathrm{C})$ and $\mathrm{mn}=$ minimum $(\mathrm{S}, \mathrm{H}, \mathrm{C})$

$H \varepsilon(0,360),(S, V) \varepsilon(0,1.0)$

\subsubsection{Color Auto-Correlogram}

The probability of determining a pixel ' $b$ ' from the pixel ' $a$ ' present at a distance $d$ are represented as $d^{\text {th }}$ entry for a pair of colors $(a, b)$. These are stored by indexing in a form of 
table and this is known as correlogram. Similarly, auto correlogram are also stored in the form of table by indexing color that gives the probability of determining a pixel 'a' from the same pixel present at a distance of $\mathrm{d}$ and is denoted by $\mathrm{d}^{\text {th }}$ entry. Spatial correlation property among the same colors and this property is represented by the auto correlogram identified. Correlation for an image 1 for a pair of images $h_{a}, h_{b}$ at a distance of $d$ is given based on formula

$\gamma_{h_{a}, h_{b}}^{d}(I m) \equiv P r_{p i_{1} \in I m_{h_{a}}, p i_{2} \in I m}\left(p i_{2} \in I m_{h_{a}}|| p i_{1}-p i_{2}=d \mid\right)$

The spatial correlation is captured by the auto correlogram based on the formula given below

$\alpha_{h}^{d}(\operatorname{Im})=\gamma_{h_{a}, h_{b}}^{d}(\mathrm{Im})$

\subsubsection{Color Co-occurrence Matrix}

A three dimensional (3D) matrix in which the first two dimensions denotes the color of any pair and the spatial distance present among the pair is denoted by the third dimension. This three dimensional matrix is known as the co-occurrence matrix. This traditional matrix similar to that of color correlogram. It is simplified in such a manner that it exhibits the total number of color pairs that is present among the neighboring pixels of an image. 4 neighbors including both horizontal and vertical are considered for a single pixel of an image.

Let $\mathrm{Im}$ represents the image of $\mathrm{N} \mathrm{x} \mathrm{M}$ size which is quantized to $\mathrm{M}$ colors, and $\mathrm{p}(\mathrm{i}, \mathrm{j})$ denotes the color of an image pixel $(i, j)$. Then, the formula for simplified CCM is given by the below equation

$H^{I m}(a, b)=\eta\left(\left(p(i, j), p\left(N_{(i, j)}\right)==(a, b)\right)\right.$

$=\alpha \sum_{i=1}^{N} \sum_{j=1}^{M} C_{a}(i, j) \sum_{\left(i^{\prime}, j^{\prime}\right) \in N_{(i, j)}} C_{b}(i, j)$

As the nearer pixel pairs are neighbors to each other, the simplified matrix thus formed is in symmetric nature. The color is quantized to 16 different colors and hence the dimension of the matrix thus formed is of size $16 \times 16$.

\subsection{Texture Features}

\subsubsection{Wavelet Features}

DWT (i.e.) Discrete Wavelet Transform was employed for calculation of mean standard deviation.

\subsubsection{NABP Features}

The proposed method utilize the novel angular binary pattern technique for the purpose of feature extraction. The method efficiently extracted the features with the following described steps.

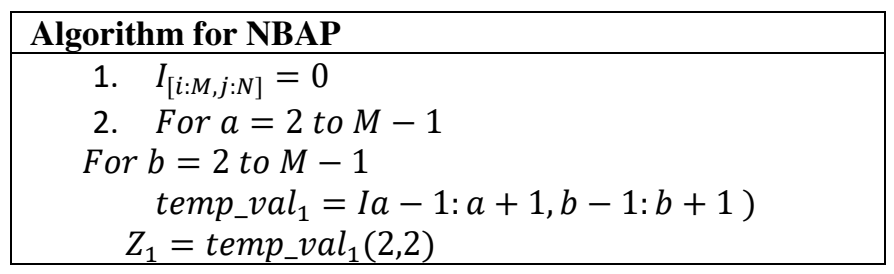

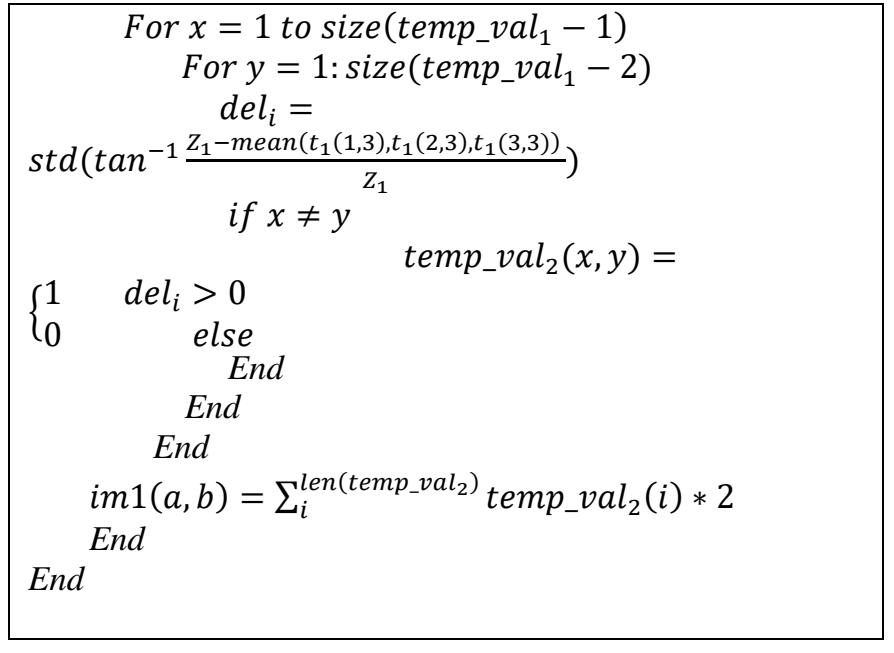

\subsubsection{GLCM Features}

Angular Second Moment,

$\mathrm{ASM}=\sum_{a=0}^{N-1} \sum_{b=0}^{N-1}\{P(a, b)\}^{2}$

Contrast,

Cont $=\sum_{i=0}^{N-1} i^{2}\left\{\sum_{a=1}^{N} \sum_{b=1}^{N} P(a, b)\right\}$,

$|a-b|=i$

Then, Inverse Difference Moment is given by

Inv. $\mathrm{DM}=\sum_{a=0}^{N-1} \sum_{b=0}^{N-1} \frac{1}{1+(a-b)^{2}} P(a, b)$

Entropy,

Ent $=\sum_{a=0}^{N-1} \sum_{b=0}^{N-1} P(a, b) \times \log (P(a, b))$

Correlation,

Cor $=\sum_{a=0}^{N-1} \sum_{b=0}^{N-1} \frac{\{a \times b\} \times P(a, b)-\left\{\mu_{p} \times \mu_{q}\right\}}{\sigma_{p} \times \sigma_{q}}$

Variance,

$\operatorname{Var}=\sum_{a=0}^{N-1} \sum_{b=0}^{N-1}(a-\mu)^{2} P(a, b)$

Mean sum,

M sum $=\sum_{a=0}^{2 N-2} a P_{p+q}(a)$

Entropy sum,

Ent $_{\text {sum }}=-\sum_{a=0}^{2 N-2} P_{p+q}(a) \log \left(P_{p+q}(a)\right)$

Entropy difference,

$E_{\text {diff. }}=-\sum_{a=0}^{N-1} P_{p+q}(a) \log \left(P_{p+q}(a)\right)$

Inertia,

Int $=\sum_{a=0}^{N-1} \sum_{b=0}^{N-1}(a-b)^{2} \times P(a, b)$

Shade of Cluster,

$C l_{s h}=\sum_{a=0}^{N-1} \sum_{b=0}^{N-1}\left\{a+b-\mu_{p}-\mu_{q}\right\}^{3} \times P(a, b)$ 
Prominence of cluster,

$C l_{P r}=\sum_{a=0}^{N-1} \sum_{b=0}^{N-1}\left\{a+b-\mu_{p}-\mu_{q}\right\}^{4} \times P(a, b)$

\subsubsection{Bit Pattern Feature}

The edges and visual patterns present in the images are characterized by the usage of Bit Pattern Feature (BPF).

\subsection{Shape Features}

\subsubsection{Object area}

It measures the object size. In object, every pixel contains the some measurement. It gives the selected Area using RoI (Region of Interest).

$\mathrm{A}(\mathrm{T})=\int S(x, y) d y d x$

Where,

$S(x, y)=1$, if the pixel contains the shape

$(x, y) \in \mathrm{T}$

$\mathrm{A}(\mathrm{T})=\sum \sum S(x, y) \Delta A$

$\Delta A$ represents area of one pixel

\subsubsection{Equivalent Diameter}

The Distance around the selected region is called the Circumference. Also, the diameter is the distance across the circle through the center. And it is calculated by

$\frac{c}{d}=\pi(5.11)$

Where,

$c$ represents circumference

$\mathrm{d}$ represents diameter

\subsubsection{Perimeter}

Perimeter defines the Distance around the boundary region. And it is calculated by

Where,

$$
\mathrm{P}=2 \mathrm{~L}+2 \mathrm{~W}
$$

$$
\begin{aligned}
& L \text { represents length } \\
& W \text { represents width }
\end{aligned}
$$

\subsection{Kernel Based Neural Network Classifier}

A segmentation network is constructed through the model of CNN (i.e.) Convolutional Neural Networks for performing segmentation of the extracted images. CNN is one among the neural networks which is utilized commonly for the classification reasons and also for performing segmentation in deep learning techniques. Some of the benefits experienced on using CNN are:

D Unlike the traditional neural networks, the $\mathrm{CNN}$ is capable of producing the learning of features.

$>$ Number of parameters utilized can be minimized through the sharing weight by CNN.

$>$ Different processes like segmentation, classification, recognition of image and enhancement of image can be performed through the usage of CNN.

The proposed architecture consists of various layers like the image input layer, convolution layer, max pooling layer, deconvolution layer and classification of image and the respective size of the layers are $128 \times 128 \times 3,64 \times 3 \times 3,2 \times 2$, $64 \times 4 \times 4$ and 2 correspondingly. Apart from that, there exists padding and strides. Padding is the output of the input image which does not correspondence with filter matrix and a stride is the total shift values during the process of convolution. Further the edge matrix value is altered to zero or valid fixed values. The second layer which is the ReLU- the Rectified Linear Unit possess the function of changing the output. If there found negative output, the ReLU is changed to zero. In this layer the size observed is $2 \times 2$ with stride 2 , padding 1 and nil output cropping.

The third layer is the pooling layer which is involved in the image matrix reduction. There exists three different kinds of pooling layer: average pooling, max pooling and sum pooling. Of those max pooling is used very commonly with $2 \times 2$ size, pooling 0 and stride 2 .

The fourth layer is the Transposed convolution layer with the size of $64 \times 4 \times 4$ with cropping output 1 and stride. The Softmax is function as the activation layer. The last layer is the pixel classification layer which possess the dimension as 2 .The segmentation methodology employed $\mathrm{CNN}$ for the following purpose. Initialization of the input image layer with predetermined format and size i.e., input size of [128 128 3].A down sampling network was created comprising the above described layers. It was followed by the creation of up sampling network comprising of ReLU and transposed convolution etc. After the creation of Softmax and classification layer, all the layers are sorted for the completion of segmentation network.

The learning rate of CNN have been found through SVM technique in the proposed paper.

\section{Performance Analysis}

The proposed system is analyzed in terms of accuracy, sensitivity, specificity, precision and recall so as to validate its efficacy. It is briefly discussed in this section.

\subsection{Performance Measures}

Performance analysis is carried out by calculating the values of various performance measures like accuracy, specificity, sensitivity, recall, precision, Jaccard coefficient, F score and missed classification.

\section{Accuracy}

Accuracy is the measurement of standard values and known as the weight arithmetic mean and it is considered as the inverse value of the precision value. The accuracy can be calculated based on the formula given below.

Accuracy $=\frac{\mathrm{TP}+\mathrm{TN}}{\mathrm{TP}+\mathrm{TN}+\mathrm{FP}+\mathrm{FN}}$

\section{Sensitivity}

Sensitivity is the measurement of the ratio of true positives to the sum of true positives and false negatives. It can also be known with the name of true positive rate. The value of sensitivity is calculated based on the following formula

True Positive rate $=\frac{\mathrm{TP}}{\mathrm{TP}+\mathrm{FN}}$

\section{Specificity}


Specificity defines the measures of the proportion of negatives that are correctly identified. It is represented as a false-positive rate or true negative rate and is the ratio of true negatives to the sum of true negative and false positive. It is calculated by using the formula mentioned below,

Specificity $=\frac{\mathrm{TN}}{\mathrm{TN}+\mathrm{FP}}$

\section{Precision}

Precision is the fraction of instances that are important in the field of information retrieval and it is the fraction of documents that are important to the request being retrieved. It is also called a high rate of prediction.

Precision value is evaluated on the basis of the formula given below,

Precision $=\left\{\frac{\{\text { relevant document } \cap \text { retrieved document }\}}{\text { retreived document }}\right\} \frac{T P}{\operatorname{ary~TP+FP}}$

\section{Recall}

A recall is the proportion of related instances that have been recovered. Therefore, both accuracy and recall are based on an understanding of significance and measurement. It is the fraction of documents relevant to the request that is successfully retrieved in the retrieval of data. It is estimated by the formula given below,

Recall $=\left\{\frac{\{\text { relevant document } \cap \text { retrieved document }\}}{\text { relevant document }}\right\}$ or $\frac{\mathrm{TP}}{\mathrm{TP}+\mathrm{FN}}$

\subsection{Results and Discussions}

The input and enhanced image are shown below in the Figure 4(a) and 4(b).

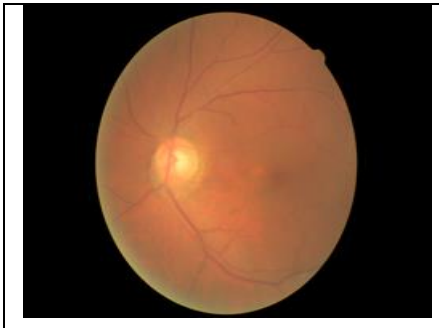

(a) Input Image

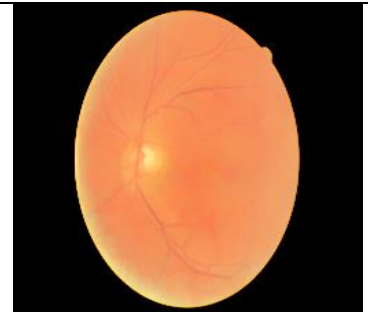

(b) Enhanced Image
Figure 4. (a) Input Image, (b) Enhanced Image
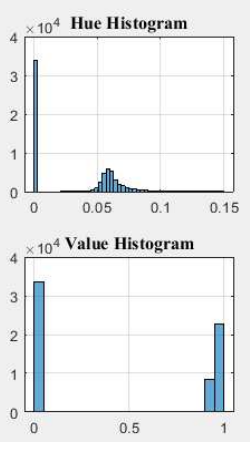

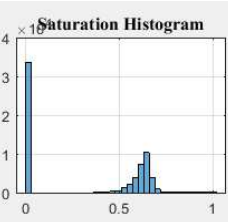

RGB Image

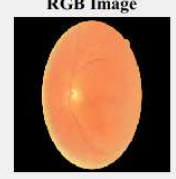

Figure 5. Image Histogram Estimat0069 on with HSV

Figure 5 denotes the histogram of an image performed with HSV. Hue Histogram, Saturation Histogram and Value Histogram are calculated separately and the RGB image obtained is also denoted in the figure.

Table 1. Investigation with the feature of color auto correlogram

\begin{tabular}{|l|l|l|l|l|l|l|l|}
\hline & 1 & 2 & 3 & 4 & 5 & 6 & 7 \\
\hline 1 & 0.9924 & 0.0393 & 0.0742 & 0 & 0.4345 & 0.5761 & 0.414 \\
\hline
\end{tabular}

The investigation of color auto correlogram is exhibited in the table 1 .

Table 2. Performance investigation with the feature of color co-occurrence

\begin{tabular}{|l|l|l|l|l|l|l|l|}
\hline & 1 & 2 & 3 & 4 & 5 & 6 & 7 \\
\hline 1 & $4.11 \mathrm{E}+03$ & 0.8125 & 0.4375 & 0.75 & 0.6875 & 0.8125 & 0.937 \\
\hline
\end{tabular}

The performance investigation of color co-occurrence is denoted in the table 2 .

Table 3. Performance investigation with the feature of Wavelet

\begin{tabular}{|l|l|l|l|l|l|l|l|}
\hline & 1 & 2 & 3 & 4 & 5 & 6 & 7 \\
\hline 1 & $1.46 \mathrm{E}+02$ & 0.0044 & 0.072 & -0.0038 & $9.96 \mathrm{E}+03$ & 70.4461 & 120.089 \\
\hline
\end{tabular}

The performance investigation of wavelet features is represented in the table 3 . 
Table 4. Performance investigation with the feature of Bit pattern

\begin{tabular}{|l|l|l|l|l|l|l|l|}
\hline & 1 & 2 & 3 & 4 & 5 & 6 & 7 \\
\hline 1 & $2.90 \mathrm{E}-03$ & 0.1392 & 0.1711 & 0.2349 & $1.42 \mathrm{E}-01$ & 0.1749 & 0.238 \\
\hline
\end{tabular}

The performance investigation of bit pattern features is represented in the table 4 .

Table 5. Performance investigation with the feature of Localized binary pattern

\begin{tabular}{|l|l|l|l|l|l|l|l|}
\hline & 1 & 2 & 3 & 4 & 5 & 6 & 7 \\
\hline 1 & $4.10 \mathrm{E}+04$ & 827 & 644 & 282 & $1.01 \mathrm{E}+03$ & 170 & 42 \\
\hline
\end{tabular}

The performance investigation of localized binary pattern features is indicated in the table 5 .

Table 6. Performance comparison of the proposed work with the existing works

\begin{tabular}{|l|l|l|l|}
\hline Performance Measures & Proposed & {$[23]$} & {$[24]$} \\
\hline Accuracy & 98.75 & 97.67 & 96.99 \\
\hline Sensitivity & 100 & 98.34 & 99.89 \\
\hline Specificity & 95 & 94.78 & 94.01 \\
\hline Precision & 98.3607 & 97.9 & 96.89 \\
\hline Recall & 100 & 99.9 & 98.78 \\
\hline F - measure & 99.1736 & 98.34 & 98.89 \\
\hline
\end{tabular}

Table 6 gives the comparison of performance measures like accuracy, sensitivity, specificity, precision, recall and F-measure of the proposed work with the existing techniques. When observing the results it is found that above discussed performance measures seemed to be more efficient for the proposed system when comparing with the existing system.

\section{Novel Kernel based CNN - Epoch Results}

The proposed novel Kernel based CNN is analyzed in terms of accuracy and loss. The analysis is carried out with different epoch. The obtained results are briefly discussed below.

When the epoch is 50 , the results are obtained as shown in the below figure 6 and figure 7 .

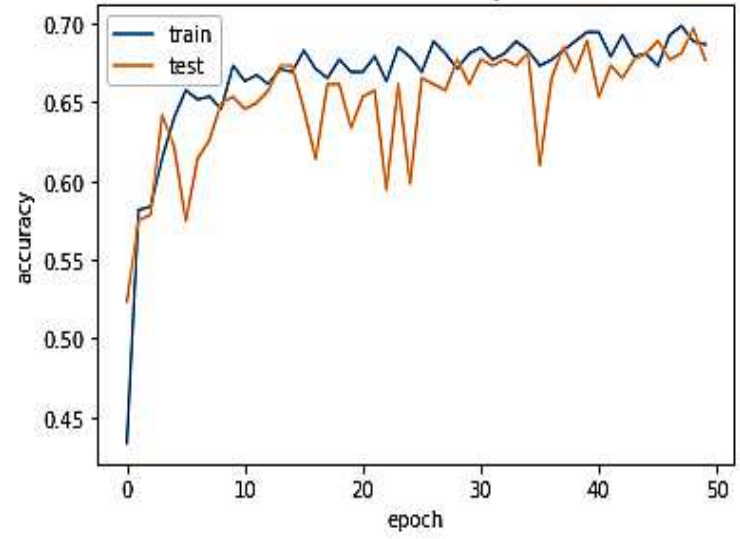

Figure 6. Results of the proposed novel Kernel based CNN in terms of accuracy when epoch -50

From the above figure 6, it is clear that the train samples has shown maximum accuracy than the test samples after each epochs. That is, the proposed novel Kernel based CNN shows that accuracy grows gradually at a steady rate thereby enhancing the accuracy of the proposed method in classifying the cataract and normal person.

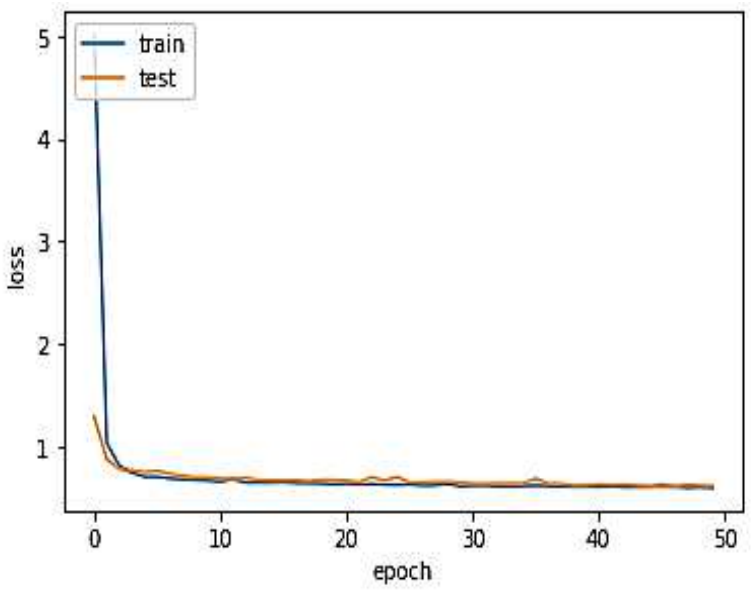

Figure 7. Results of the proposed novel Kernel based CNN in terms of loss when epoch -50

From the above figure 7, it is clear that the train and test samples decreases at a gradual rate. This indicates that the proposed 
novel Kernel based CNN decreases loss gradually. This eventually increases the system efficiency.

Table 7. Classification of cataract and normal person by the proposed novel Kernel based CNN when epoch is 50

\begin{tabular}{|l|c|c|}
\hline \multicolumn{3}{|c|}{ Epoch 50 } \\
\hline & Cataract & Normal \\
\hline Cataract & 80 & 20 \\
\hline Normal & 20 & 250 \\
\hline
\end{tabular}

From the above table 7, the above confusion matrix shows that 80 people are found to have cataract and 250 people are found to be normal. This classification is performed correctly. While, the misclassification is found to be 20 . Here the classification rate is higher than the misclassification rate at a particular extent when epoch is 50 . This reveals the efficiency of the proposed methodology.

Similarly, when the epoch is 100 , the results are obtained as shown in the below figure 8 and figure 9.

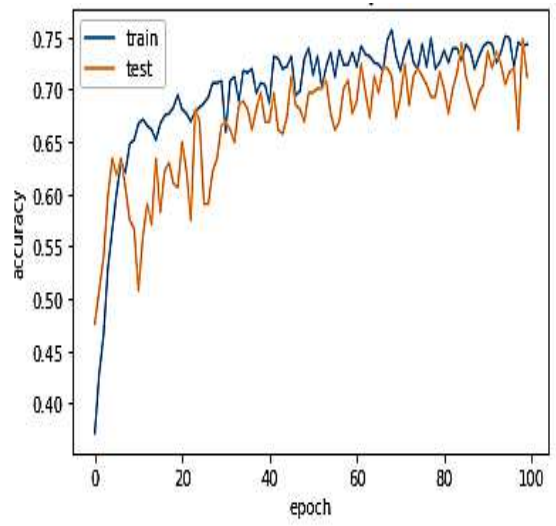

Figure 8. Results of the proposed novel Kernel based CNN in terms of accuracy when epoch -100

From the above figure. 8 , it is clear that the train samples has shown maximum accuracy than the test samples after each epochs. That is, the proposed novel Kernel based CNN shows that accuracy grows gradually at a steady rate at the $20^{\text {th }}$ epoch thereby enhancing the accuracy of the proposed method in classifying the cataract and normal person.

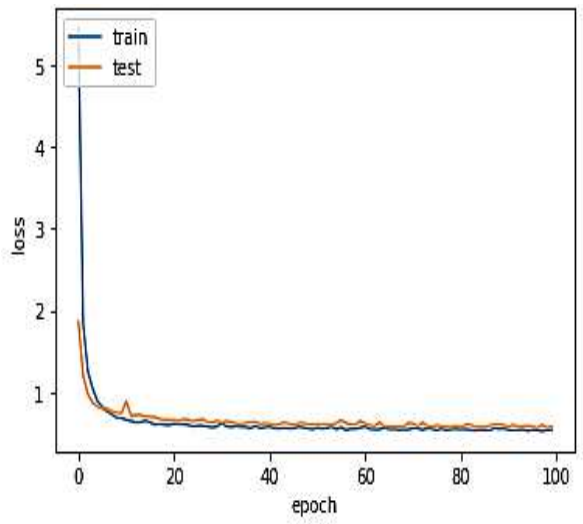

Figure 9. Results of the proposed novel Kernel based CNN in terms of loss when epoch -100

From the above figure 9 , it is clear that the train and test samples decreases at a gradual rate. This indicates that the proposed novel Kernel based CNN decreases loss gradually which eventually increases the system efficiency.

Table 8. Classification of cataract and normal person by the proposed novel Kernel based CNN when epoch is 100

\begin{tabular}{|l|r|r|}
\hline \multicolumn{3}{|c|}{ Epoch 100 } \\
\hline & Cataract & Normal \\
\hline Cataract & 85 & 15 \\
\hline Normal & 17 & 253 \\
\hline
\end{tabular}

From the above table 8 , the above confusion matrix shows that 85 people are found to have cataract and 253 people are found to be normal. This classification is performed correctly. On the other hand, the 15 cataract person is misclassified as normal and 17 normal people are misclassified as cataract. Here the classification rate is higher than the misclassification rate at a particular extent when epoch is 100 . This reveals the efficiency of the proposed methodology.

When the epoch is 150 , the results are obtained as shown in the below figure 10 and figure 11.

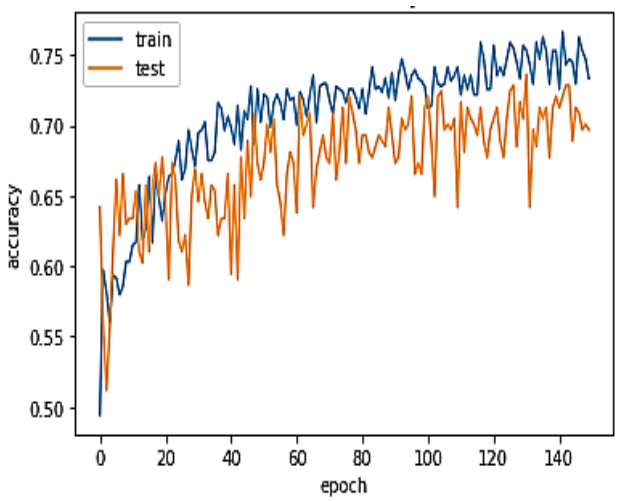

Figure 10. Results of the proposed novel Kernel based CNN in terms of accuracy when epoch -150 
The above figure 10 shows that the train samples has shown maximum accuracy than the test samples after each epochs. That is, the proposed novel Kernel based CNN explores that accuracy grows gradually at a steady rate at the $20^{\text {th }}$ epoch thereby increasing the classification accuracy of the proposed novel Kernel based CNN.

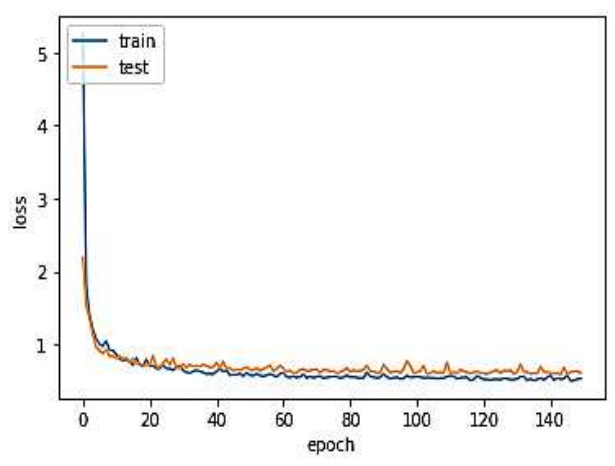

Figure 11. Results of the proposed novel Kernel based CNN in terms of loss when epoch -150

From the above figure 11 , it is clear that the train and test samples decreases gradually. This indicates that the proposed novel Kernel based CNN decreases loss gradually which eventually increases the system efficiency.

Table 9. Classification of cataract and normal person by the proposed novel Kernel based CNN when epoch is 150

\begin{tabular}{|l|r|r|}
\hline \multicolumn{3}{|c|}{ Epoch 150 } \\
\hline & Cataract & Normal \\
\hline Cataract & 89 & 11 \\
\hline Normal & 15 & 255 \\
\hline
\end{tabular}

From the above table 9, the above confusion matrix shows that 89 people are found to have cataract and 255 people are found to be normal. This classification is performed correctly. On the other hand, the 11 cataract individuals are misclassified as normal and 15 normal people are misclassified as cataract. Here the classification rate is higher than the misclassification rate at a particular extent when epoch is 150 . This reveals the efficiency of the proposed methodology.

In addition, the proposed system is compared and analyzed with the existing systems in terms of accuracy. It is shown in the below table 5 .

Table 10. Comparative analysis of the proposed and existing systems [25] in terms of accuracy

\begin{tabular}{|l|l|}
\hline Method & Accuracy \\
\hline Ran & 0.9069 \\
\hline $\mathrm{Li}$ & 0.877 \\
\hline $\mathrm{Xu}$ & 0.8624 \\
\hline
\end{tabular}

\begin{tabular}{|l|l|}
\hline $\mathrm{Li}$ & 0.9493 \\
\hline proposed method & 0.9739 \\
\hline
\end{tabular}

From the above table 10, it if found that the proposed system shows 0.9739 as its accuracy rate. It is higher than the existing systems [25] which explores the efficacy of the recommended methodology.

\section{CONCLUSION}

Cataract is a condition of opacity in the lenticular regions which usually leads to poor visual interpretation of the object or any entity being viewed. Therefore, timely cataract detection is considered relevant and can even contribute to the prevention of loss of fighting that could occur if the cataract remains untreated. In this paper, cataract disease detection is performed based on the technique of image processing. Color features, texture features and shape features are extracted separately. A Novel Angular Binary Pattern (NABP) is utilized in this study for the extraction of relevant texture features. And after feature extraction, the images are subjected to classification by implementing a novel Kernel-based Convolutional Neural Networks. A comparison is made with existing works for the proposed work and based on the results collected it can be seen that the proposed work shows improved performance than the existing systems in cataract classification.

\section{REFERENCES}

[1] L. Cao, H. Li, Y. Zhang, L. Zhang, and L. Xu, "Hierarchical method for cataract grading based on retinal images using improved Haar wavelet," Information Fusion, vol. 53, pp. 196-208, 2020.

[2] L. Xiong, H. Li, and L. Xu, "An approach to evaluate blurriness in retinal images with vitreous opacity for cataract diagnosis," Journal of healthcare engineering, vol. 2017, 2017.

[3] Y.-C. Liu, M. Wilkins, T. Kim, B. Malyugin, and J. S. Mehta, "Cataracts," The Lancet, vol. 390, pp. 600-612, 2017.

[4] J.-J. Yang, J. Li, R. Shen, Y. Zeng, J. He, J. Bi, et al., "Exploiting ensemble learning for automatic cataract detection and grading," Computer methods and programs in biomedicine, vol. 124, pp. 4557, 2016.

[5] H. E. Gali, R. Sella, and N. A. Afshari, "Cataract grading systems: a review of past and present," Current opinion in ophthalmology, vol. 30, pp. 13-18, 2019.

[6] M. K. Sreejaya, A. Vijayan, A. Krishnan, and D. Sreedharan, "Various cataract detection methods-a survey," Int Res J Eng Technol, vol. 4, pp. 1517-1519, 2017.

[7] H. R. Tawfik, R. A. Birry, and A. A. Saad, "Early Recognition and Grading of Cataract Using a Combined Log Gabor/Discrete Wavelet Transform with ANN and SVM," International Journal of Computer and Information Engineering, vol. 12, pp. 1038-1043, 2018.

[8] B. R. Kumar and M. Shimna, "Recent Approaches for Automatic Cataract Detection Analysis Using Image Processing," Journal of Network Communications and Emerging Technologies (JNCET) www. jncet. org, vol. 7, 2017.

[9] M. K. Sreejaya, A. Vijayan, A. Krishnan, and D. Sreedharan, "VARIOUS CATARACT DETECTION METHODS-A SURVEY," 2017.

[10] M. Manchalwar and K. Warhade, "Detection of Cataract and Conjunctivitis Disease Using Histogram of Oriented Gradient," International Journal of Engineering and Technology (IJET), 2017. [11] A. Imran, J. Li, Y. Pei, J.-J. Yang, and Q. Wang, "Comparative Analysis of Vessel Segmentation Techniques in Retinal Images," IEEE Access, vol. 7, pp. 114862-114887, 2019.

[12] I. Shaheen and A. Tariq, "Survey Analysis of Automatic Detection and Grading of Cataract Using Different Imaging Modalities," in 
Applications of Intelligent Technologies in Healthcare, ed: Springer, 2019, pp. 35-45.

[13] M. Kim, Y. Eom, J. S. Song, and H. M. Kim, "Effect of cataract grade according to wide-field fundus images on measurement of macular thickness in cataract patients," Korean Journal of Ophthalmology, vol. 32, pp. 172-181, 2018.

[14] S. Kolhe and S. K. Guru, "Remote Automated Cataract Detection System Based on Fundus Images," International Journal of Innovative Research in Science, Engineering and Technology, vol. 5, p. 23, 2016.

[15] D. Podkowinski, E. Sharian Varnousfaderani, C. Simader, H Bogunovic, A.-M. Philip, B. S. Gerendas, et al., "Impact of B-Scan averaging on Spectralis optical coherence tomography image quality before and after cataract surgery," Journal of ophthalmology, vol. 2017, 2017.

[16] I. Jindal, P. Gupta, and A. Goyal, "Cataract Detection using Digital Image Processing," in 2019 Global Conference for Advancement in Technology (GCAT), 2019, pp. 1-4.

[17] J. Kaur, P. Sinha, R. Shukla, and V. Tiwari, "Automatic Cataract Detection Using Haar Cascade Classifier," in Data Intelligence and Cognitive Informatics, ed: Springer, 2021, pp. 543-556.

[18] X. Xu, L. Zhang, J. Li, Y. Guan, and L. Zhang, "A hybrid globallocal representation CNN model for automatic cataract grading," IEEE journal of biomedical and health informatics, vol. 24, pp. 556$567,2019$.

[19] M. Yusuf, S. Theophilous, J. Adejoke, and A. B. Hassan, "WebBased Cataract Detection System Using Deep Convolutional Neural Network," in 2019 2nd International Conference of the IEEE Nigeria Computer Chapter (NigeriaComputConf), 2019, pp. 1-7.

[20] I. Weni, P. E. P. Utomo, B. F. Hutabarat, and M. Alfalah, "Detection of Cataract Based on Image Features Using Convolutional Neural Networks," IJCCS (Indonesian Journal of Computing and Cybernetics Systems), vol. 15, pp. 75-86.

[21] V. Holennavar and P. Kumar, "Instant Detection of Cataracts."

[22] S. Agarwal, M. Kumar, S. K. Jangir, and C. Sharma, "ComputerAided Cataract Detection Using MLP and SVM," Artificial Intelligence and Global Society: Impact and Practices, p. 103, 2021.

[23] E. Ouabida, A. Essadike, and A. Bouzid, "Automated segmentation of ophthalmological images by an optical based approach for early detection of eye tumor growing," Physica Medica, vol. 48, pp. 3746, 2018.

[24] A. Daruich, A. Matet, and F. L. Munier, "Cataract development in children with Coats disease: risk factors and outcome," Journal of American Association for Pediatric Ophthalmology and Strabismus, vol. 22, pp. 44-49, 2018.

[25] A. Imran, J. Li, Y. Pei, F. Akhtar, T. Mahmood, and L. Zhang, "Fundus image-based cataract classification using a hybrid convolutional and recurrent neural network," The Visual Computer, pp. 1-11, 2020 . 
Figures

\section{Normal Eye}

\section{Cataract Eye}
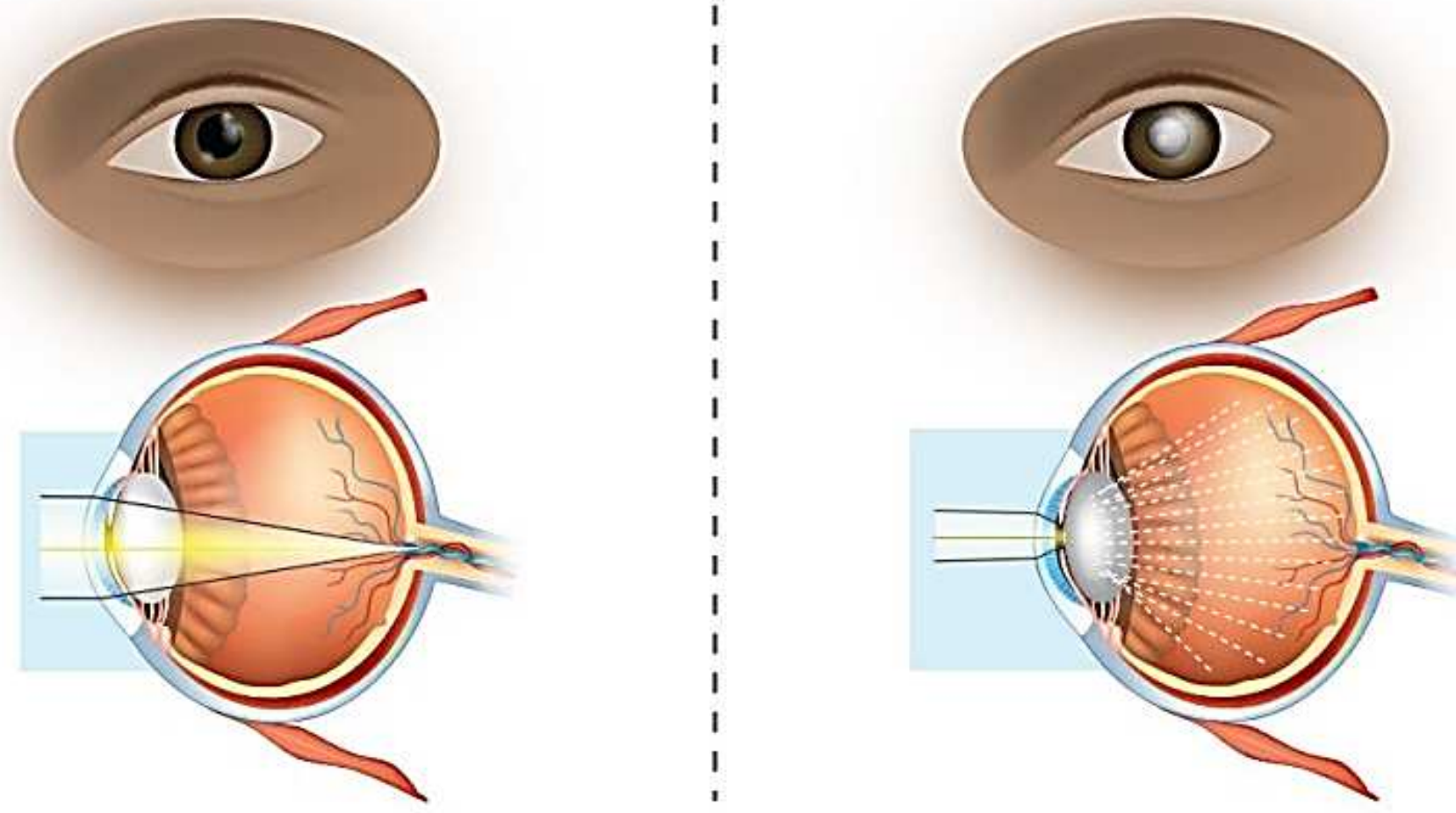

Figure 1

Difference between normal and cataract eye<smiles>OO[14CH2]OO</smiles>

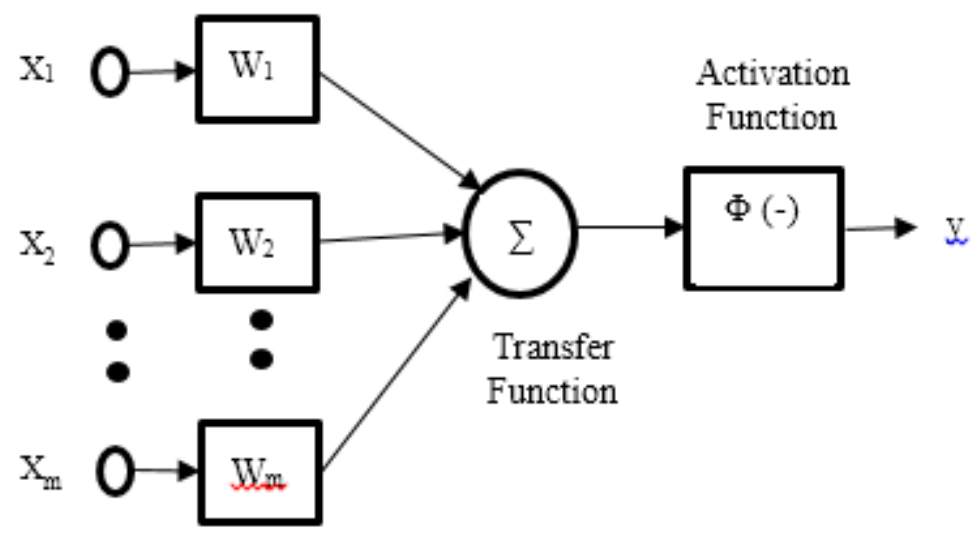

Figure 2

Kernel based CNN architecture 


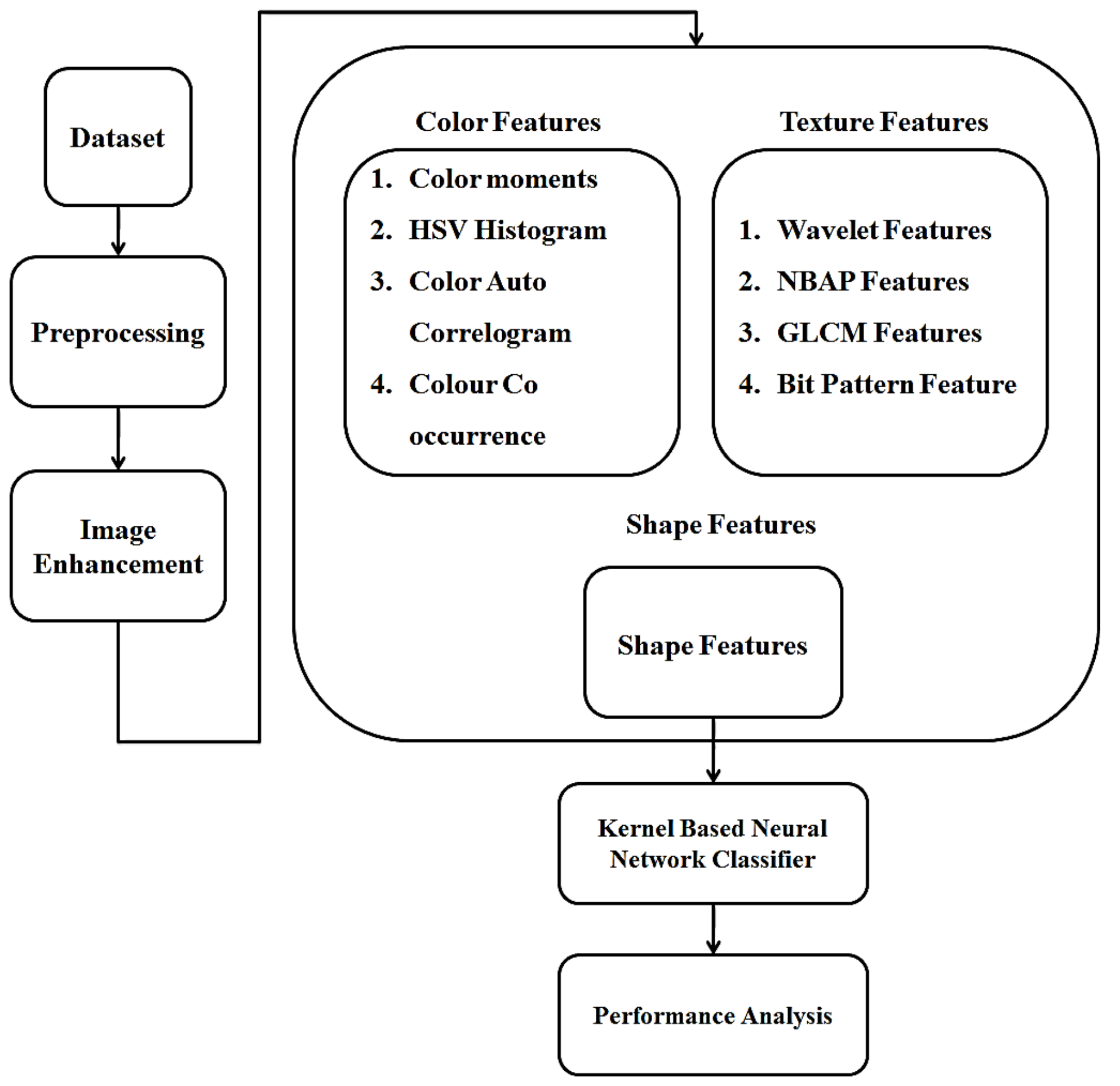

Figure 3

Overall Flow of the proposed work 


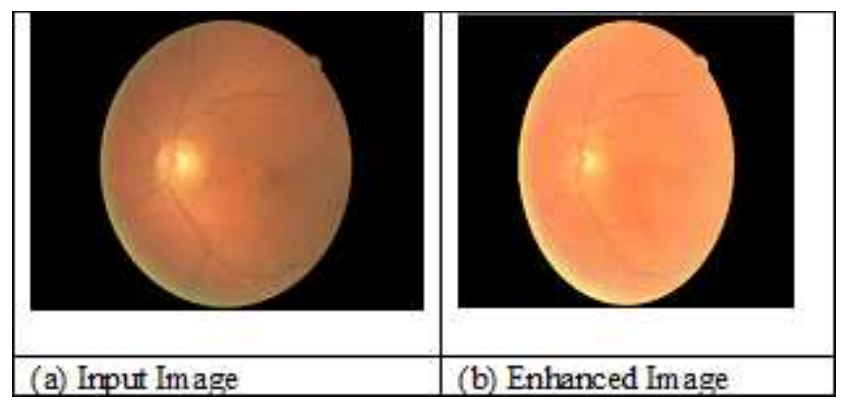

Figure 4

(a) Input Image, (b) Enhanced Image

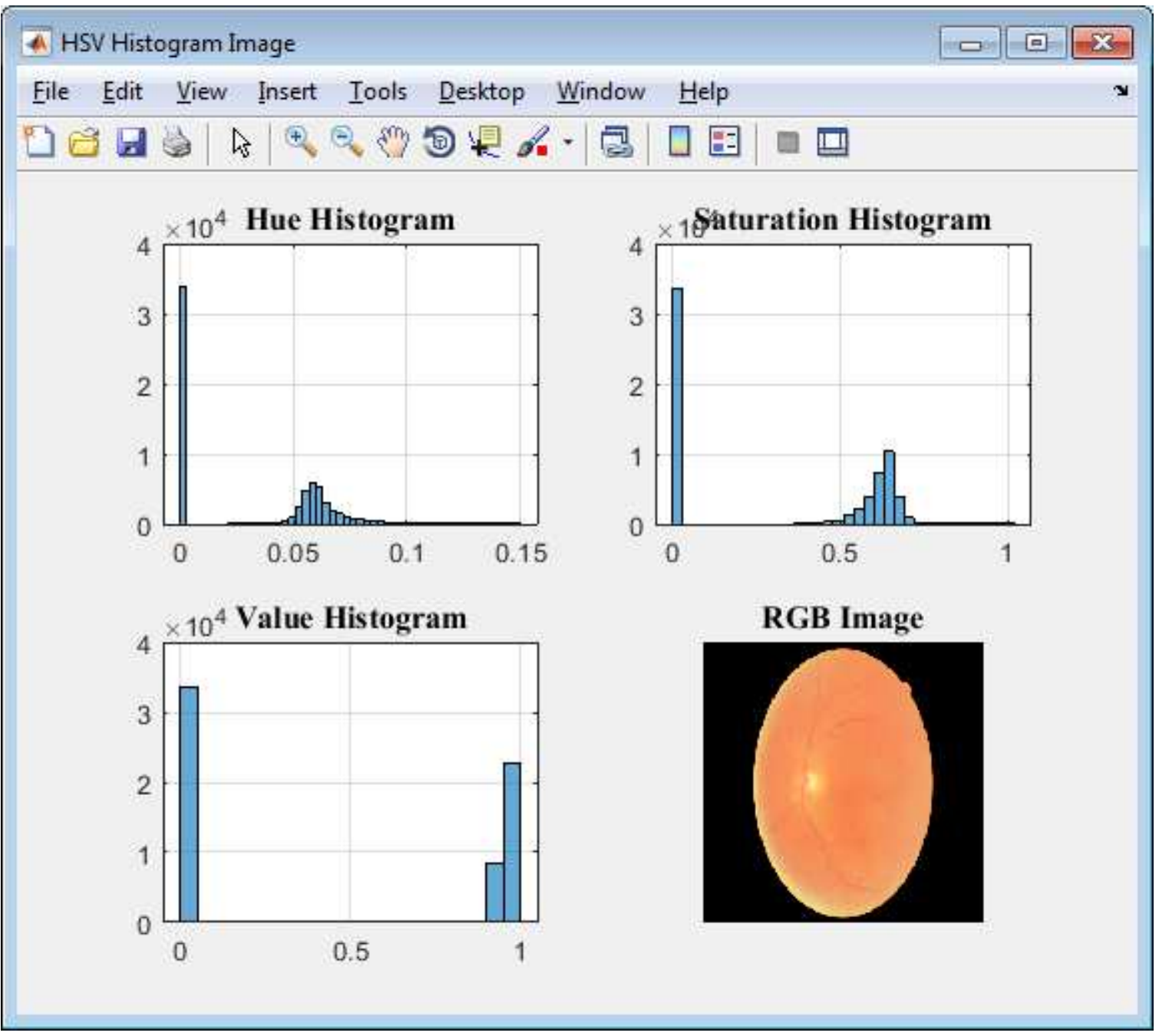

Figure 5

Image Histogram Estimat0069 on with HSV 


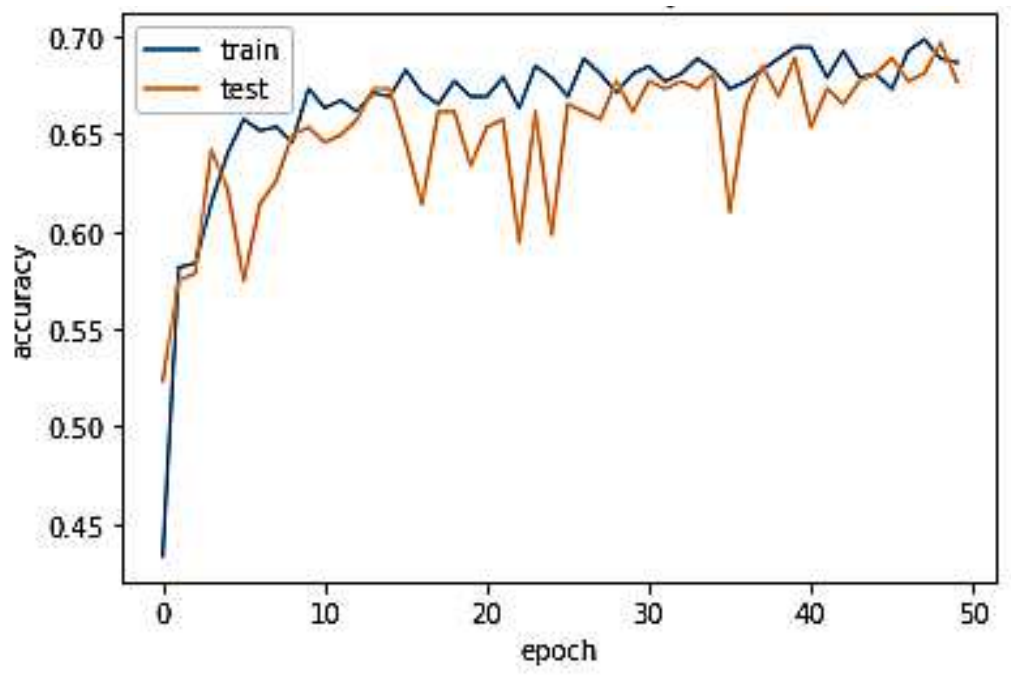

Figure 6

Results of the proposed novel Kernel based CNN in terms of accuracy when epoch - 50

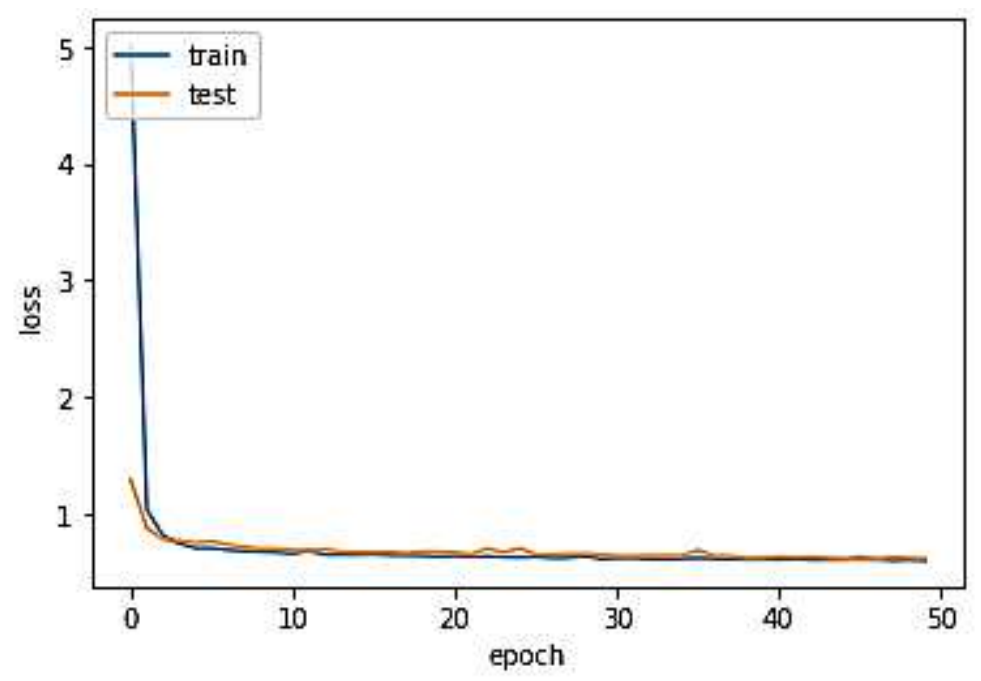

Figure 7

Results of the proposed novel Kernel based CNN in terms of loss when epoch - 50

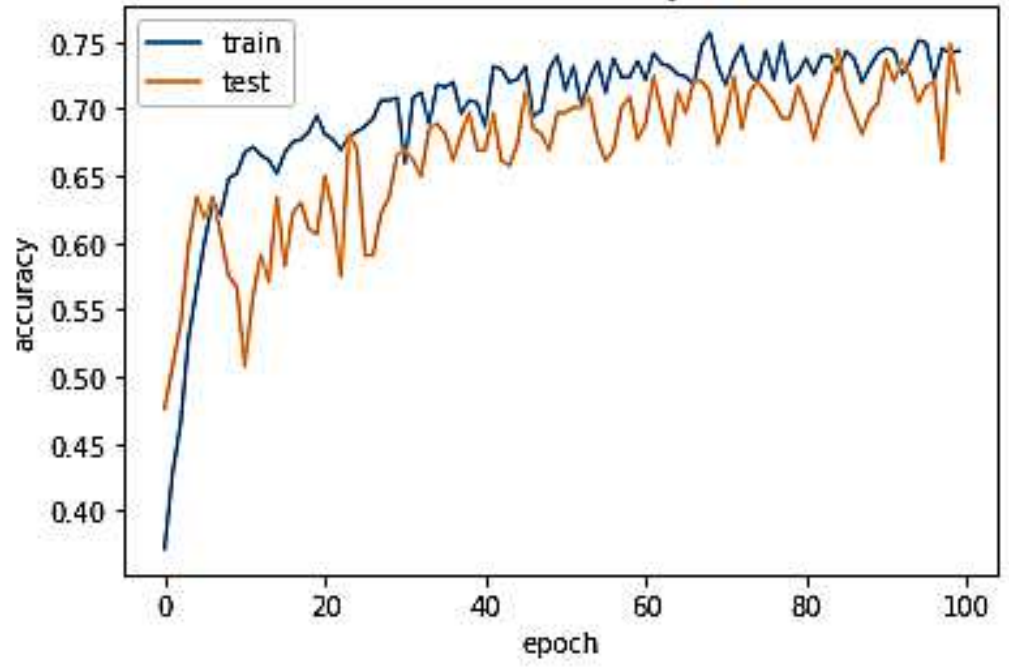


Figure 8

Results of the proposed novel Kernel based CNN in terms of accuracy when epoch - 100

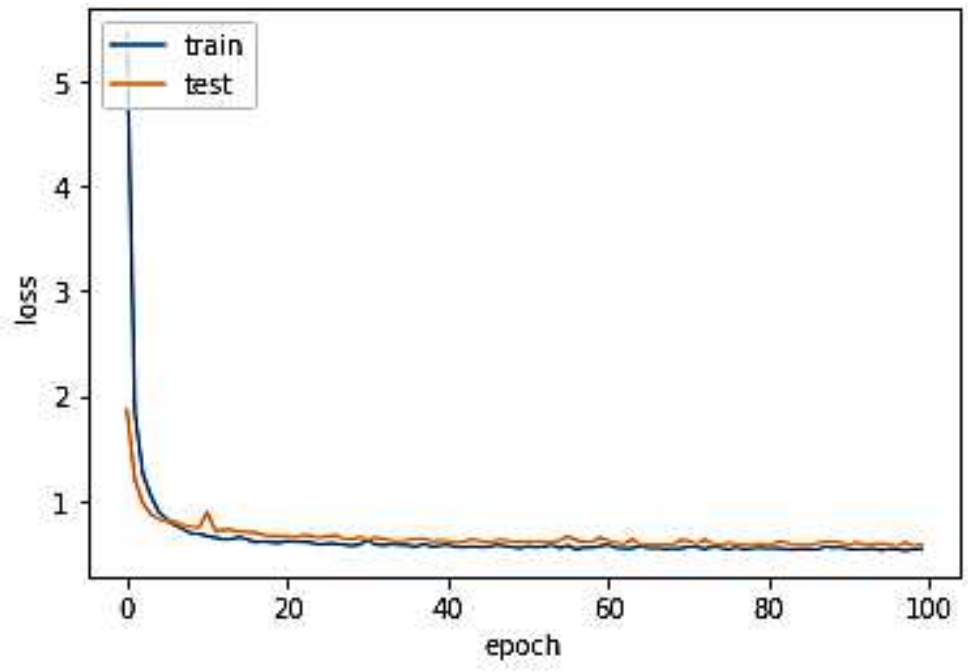

Figure 9

Results of the proposed novel Kernel based CNN in terms of loss when epoch - 100

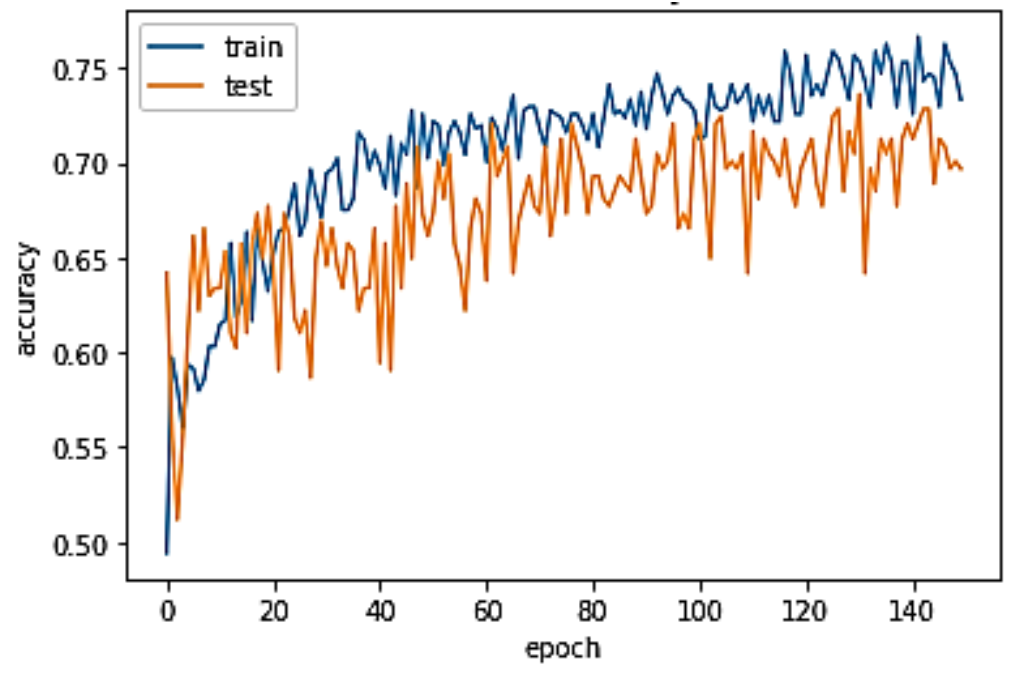

Figure 10

Results of the proposed novel Kernel based CNN in terms of accuracy when epoch - 150 


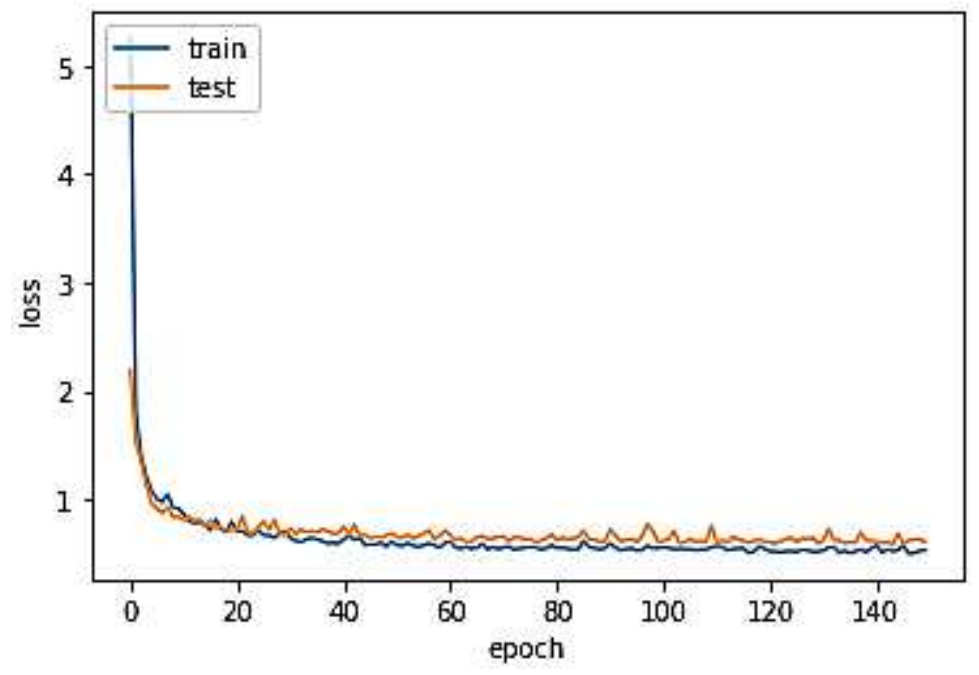

Figure 11

Results of the proposed novel Kernel based CNN in terms of loss when epoch - 150 